\title{
Article
}

\section{Synthesis of Bulk Zeolite by Modified Hydrothermal Process and Its Characterization}

\author{
Hidezumi Nagata, Takashi Kubo, and *Atsushi Nakahira \\ Osaka Prefecture University, 1-1 Galuencho, Naka-ku, Sakai, Osaka 599-8531, Japan \\ Takamasa Onoki \\ Tokyo Institute of Technology, 4259, Nagatsuta-cho, Midori-ku, Yokohama 226-8503, Japan \\ Yuki Yamasaki \\ Hosei University, 4342, Aihara-cho, Machida, Tokyo 194-0298, Japan
}

(Manuscript submitted March 31, 2007; accepted May 31, 2007)

\begin{abstract}
Fabrication of bulk zeolite such as silver ion exchanged Y-zeolites was attempted by the modified hydrothermal processing. The present synthetic processing was modified from thermal hot-pressing developed by Yamasaki. Using this process, we were successful for the dense bulky silver ion exchanged Y-zeolite. In this study the microstructure of bulk FAU zeolite were observed by FE-SEM, XRD and XAFS. Also, the relation of microstructure and properties were discussed.
\end{abstract}

Key words: ion exchange, zeolite, FAU-type, bulk, hydrothermal hot-pressing

\section{Introduction}

Zeolites with possess well-defined three-dimensional (3D) micropores have been much attention for applications as catalysis carrier, gas separation, adsorption and ion exchanger. Especially, $\mathrm{Ag}^{+}$ion-exchanged zeolites are quite unique materials with actions such as photochemical/thermal cleavage of water into $\mathrm{H}_{2}$ and $\mathrm{O}_{2}$ [1], photo-oxygen production from water [2], and photocatalytic decomposition of NO [3, 4]. Silver ions can be reduced intrazeolitically by heating [5, 6], by reaction with reducing agents [7], or by sorption of metal atoms [8]. Tsutsumi and Takahashi reported that the $\mathrm{Ag}^{+}$ions in zeolite $\mathrm{Y}$ can be reduced to bulk clusters of $\mathrm{Ag}^{0}$ after treatment with alcohol and alkylbenzene above $300{ }^{\circ} \mathrm{C}$ [9]. It was, however, restricted the applications because zeolites are obtained as a powder only and was not able to obtain a solidified body except for small single crystalline zeolite [10]. Thus, many researches have tried to make powder consolidation and thin film for expansion of applications of zeolite. However, there is still a problem that microporous structure was not able to be retained for bulky or filmy zeolite synthesized in conventional method, because they contain a lot of parts of amorphous and portion of micropores structure were filled with amorphous phase. Therefore, practical use of zeolites has not been achieved yet.

Our group has proposed to obtain solidified zeolite bulk using Hydrothermal Hot-pressing (HHP) method. The HHP technique is a method by which hard solid bodies of powders can be produced in a short time and at a relatively low temperature under saturated vapor pressure [11]. Nakahira et al. reported that densified and translucent bulky Y-zeolite was synthesized using HHP technique [12]. Hence, the purpose in this paper is to attempt to synthesize $\mathrm{Ag}^{+}$ion exchanged Y-zeolite (refer as $\mathrm{Ag}$-Y-zeolite) by this HHP technique and reveal the 
microstructure of bulky Ag-Y-zeolite observed by FE-SEM. Moreover, the local structure of the $\mathrm{Ag}^{+}$ion in Ag-Y-zeolite bulk compared to Ag-Y-zeolite powder observed by XAFS.

\section{Experimental}

\subsection{Ion Exchange Reaction}

Ag-Y-zeolite sample was prepared by conventional ion-exchange technique using $1.0 \times 10^{-2} \mathrm{MgNO}_{3}$ solution and Na-Y-zeolite (HSZ-320NAA, particle size; 0.3 to $0.4 \mu \mathrm{m}, \mathrm{SiO}_{2} / \mathrm{Al}_{2} \mathrm{O}_{3}=5.5$, specific surface area; 860 $\mathrm{m}^{2} / \mathrm{g}$, Tosoh Co.) under stirring at $70{ }^{\circ} \mathrm{C}$ for $10 \mathrm{~h}$. After filtration, the powder was dried at $323 \mathrm{~K}$ overnight. When the zeolite is in the exchange medium, equilibrium is established;

$$
\left.\mathrm{Na}^{+}(\text {zeolite })+\mathrm{Ag}^{+}(\mathrm{aq}) \gtrless \mathrm{Ag}^{+} \text {(zeolite }\right)+\mathrm{Na}^{+}(\mathrm{aq})
$$

\subsection{Hydrothermal hot-pressing}

Ion exchanged Ag-Y-zeolite powder were used as a starting powder. Figure 1 showed an autoclave for HHP in this study. Ag-Y-zeolite powder $(0.75 \mathrm{~g})$ was added to $20 \mathrm{wt} \%$ of various concentration of $\mathrm{NaOH}$ solution, which was placed into the autoclave. This autoclave containing sample was heated at $423 \mathrm{~K}$ at the rate of $10 \mathrm{~K} / \mathrm{min}$ under the pressure with $40 \mathrm{MPa}$ for $2 \mathrm{~h}$. After HHP treatment, the autoclave was cooled to a room temperature and the product removed from the autoclave. Products treated by HHP method were washed and dried at $323 \mathrm{~K}$ for more than $12 \mathrm{~h}$. Some Ag-Y-zeolite bulks were calcined in $\mathrm{H}_{2}$ at $773 \mathrm{~K}$ for $2 \mathrm{~h}$.

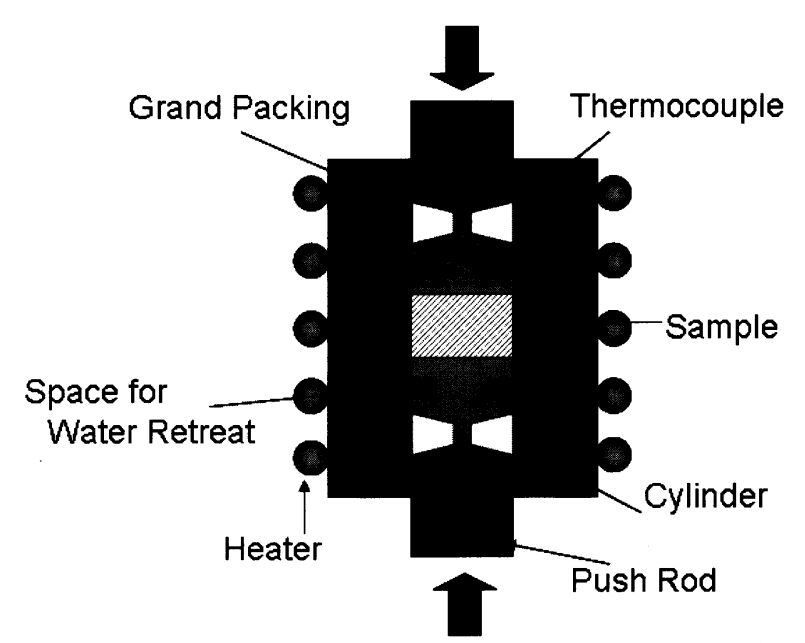

Fig. 1 Autoclave for hydrothermal hot-pressing.

\subsection{Characterizations}

Products prepared by HHP were identified by powder X-ray diffractometry (XRD, RINT2500, Rigaku) with $\mathrm{CuK} \alpha$ radiation, operating at $40 \mathrm{kV}$ and $50 \mathrm{~mA}$. The microstructures of products were observed by field emission scanning electron microscopy (FE-SEM, S-4500, HITACHI). Nitrogen adsorption isotherms at $77 \mathrm{~K}$ were obtained by automatic gas adsorption measure apparatus (BELSORP18PLUS-SPL, Japan BEL). Some pieces of product were pretreated at $403 \mathrm{~K}$ for $12 \mathrm{~h}$. Specific surface area of products was calculated by a Langmuir plot. The Ag K-edge XAFS spectra were obtained in the fluorescence mode at $298 \mathrm{~K}$.

\section{Results and Discussion}

The effect of $\mathrm{NaOH}$ concentration on the microstructure of Ag-Y-zeolite sample by HHP was examined. Under various synthetic conditions of HHP, Ag-Y-zeolite bulks were obtained. Strong Ag-Y-zeolite bulks obtained with $5 \mathrm{M} \mathrm{NaOH}$ solution on HHP, which bulk density was approximately $1.80 \mathrm{~g} / \mathrm{cm}^{3}$. Although bulk density of Ag-Y-zeolite bulk with $1 \mathrm{M} \mathrm{NaOH}$ solution was $1.03 \mathrm{~g} / \mathrm{cm}^{3}$, it is likely suggested that the addition of low concentration $\mathrm{NaOH}$ solution could not led to appropriate hydrothermal condition. Thus, optimum $\mathrm{NaOH}$ concentrations on HHP existed for preparing dense Ag-Y-zeolite bulks.

Figure 2 shows SEM images of HHP-treated samples with 1 or $5 \mathrm{M} \mathrm{NaOH}$ solution before/after heat treatment in $\mathrm{H}_{2}$. SEM observation indicated that $\mathrm{HHP}$-treated samples with $1 \mathrm{M} \mathrm{NaOH}$ before/after heat treatment in $\mathrm{H}_{2}$ showed some voids and they were porous and not densified. On the other hand, HHP-treated samples with 5 $M \mathrm{NaOH}$ before/after heat treatment in $\mathrm{H}_{2}$ showed a very dense bulk surface without defects such as pinholes and 

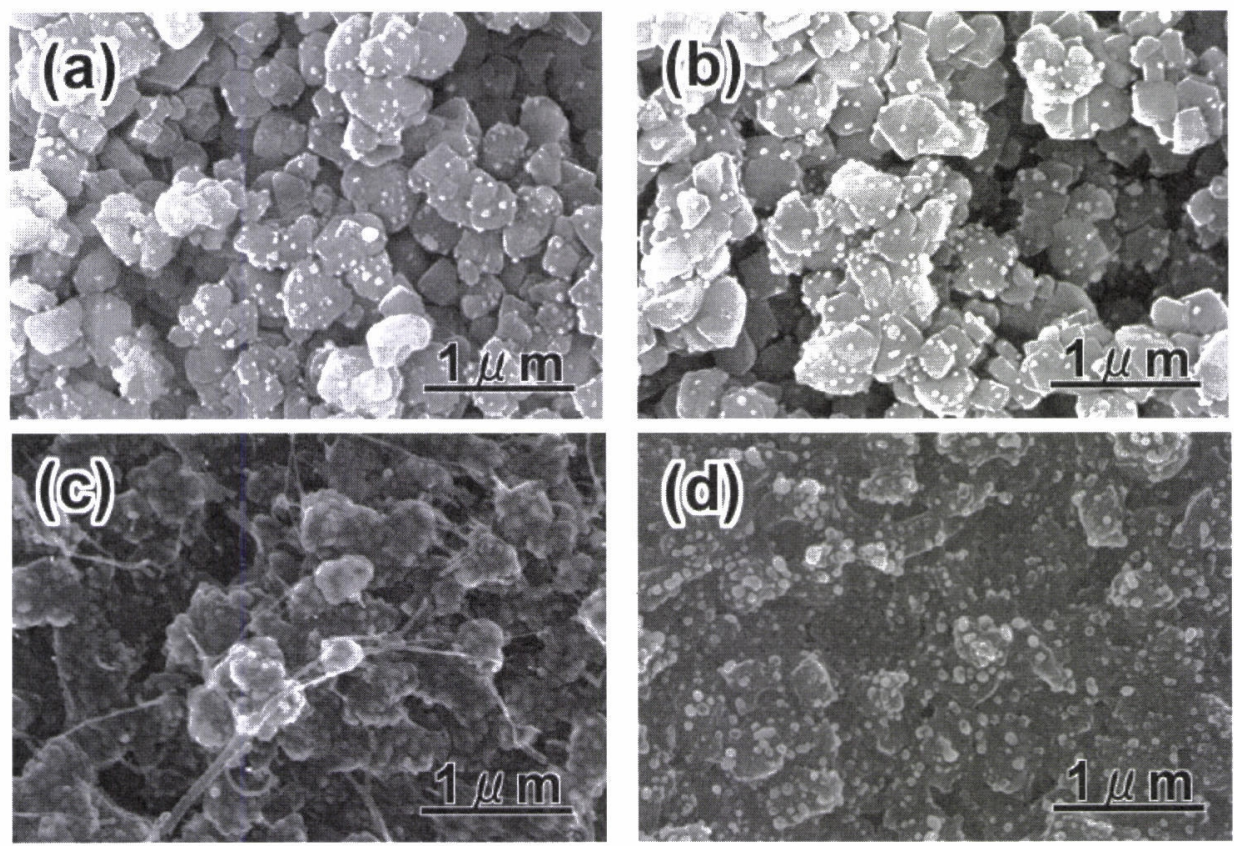

Fig. 2 SEM images of HHP-treated samples with $1 M \mathrm{NaOH}$ (a) before, (b) after heat treatment in $\mathrm{H}_{2}$, and with $5 \mathrm{MNaOH}$ (c) before, (d) after heat treatment in $\mathrm{H}_{2}$.

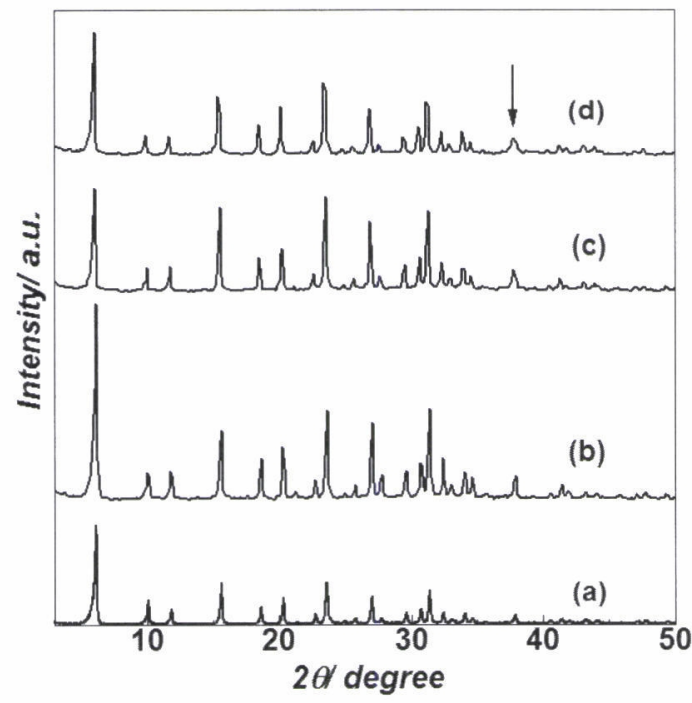

Fig. 3 XRD patterns of (a) Y-zeolite powder, (b) Ag-Y-zeolite powder, (c) Ag-Y-zeolite powder calcined $500{ }^{\circ} \mathrm{C}$ in $\mathrm{H}_{2}$, (d) Ag-Y-zeolite HHP bulk calcined $500{ }^{\circ} \mathrm{C}$ in $\mathrm{H}_{2}$.

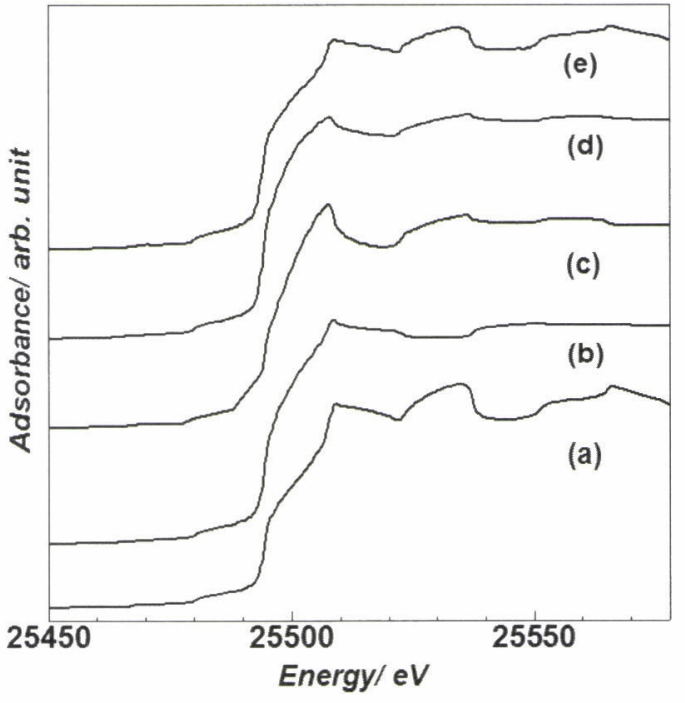

Fig. 4 XANES spectra of (a) Ag foil, (b) $\mathrm{Ag}_{2} \mathrm{O}$, (c) Ag-Y-zeolite powder, (d) Ag-Y-zeolite powder calcined $500{ }^{\circ} \mathrm{C}$ in $\mathrm{H}_{2}$, (e) Ag-Y-zeolite HHP bulk calcined $500{ }^{\circ} \mathrm{C}$ in $\mathrm{H}_{2}$.

cracks. It is founded that the successful synthesis condition for preparing the densest bulk HHP-treated sample is done under $5 \mathrm{M} \mathrm{NaOH}$ solutions. Thus, this dense and pinhole-free microstructure consisting of fine zeolite crystals are successfully obtained.

Ag-Y-zeolite samples were evaluated by XRD, as shown in Fig. 3. The XRD results showed that all products were identified as Y-zeolite. The peak width of Ag-Y-zeolite HHP bulk calcined $500{ }^{\circ} \mathrm{C}$ in $\mathrm{H}_{2}$ around 2 
$\theta=38^{\circ}$ (marked as $\downarrow$ ) was extended slightly. This is thought to be due to overlapping of the identified peaks of Y-zeolite and $\mathrm{Ag}$ metal, which indicated to produce $\mathrm{Ag}$ metal by the reduction of $\mathrm{Ag}^{+}$ions. Specific surface area of Ag-Y-zeolite powder, and Ag-Y-zeolite bulk prepared by $\mathrm{HHP}$ with 1 and $5 \mathrm{M} \mathrm{NaOH}$ solution were $810 \mathrm{~m}^{2} / \mathrm{g}, 605$ $\mathrm{m}^{2} / \mathrm{g}$ and $695 \mathrm{~m}^{2} / \mathrm{g}$, respectively. This result showed that a high specific surface area of dense bulk zeolite solidified upon HHP treatment was comparable with that of the Ag-Y-zeolite powder as a starting material.

Fig. 4 shows the XANES spectra of Ag-Y-Zeolite powder and bulk and reference materials such as $\mathrm{Ag}_{2} \mathrm{O}$ and $\mathrm{Ag}$ foil. Shapes and positions of the bands due to multiple scattering in XANES spectrum of the (c) Ag-Y-zeolite powder and (d) Ag-Y-Zeolite powder calcined $500{ }^{\circ} \mathrm{C}$ in $\mathrm{H}_{2}$ were quite different from those of (a) $\mathrm{Ag}$ foil and (b) $\mathrm{Ag}_{2} \mathrm{O}$. This indicates that the large clusters of $\mathrm{Ag}_{2} \mathrm{O}$ or $\mathrm{Ag}$ metal are not formed within zeolite cavities. XANE spectrum of (e) Ag-Y-zeolite HHP bulk calcined $500{ }^{\circ} \mathrm{C}$ in $\mathrm{H}_{2}$ was similar to that of (a) $\mathrm{Ag}$ foil, which implies that there are silver metal due to reduction of $\mathrm{Ag}^{+}$ions.

\section{Conclusion}

In this paper, solidified bulky Ag-Y-zeolite could be successfully synthesized by HHP treatment for short term. It was founded that the dense Ag-Y-zeolite by HHP was obtained under $5 \mathrm{MNaOH}$ solution as solvent. Solidified Ag-Y-zeolite bulk by HHP with $5 \mathrm{M} \mathrm{NaOH}$ solution was identified as Y-zeolite polycrystalline and had significantly large surface area over $600 \mathrm{~m}^{2} / \mathrm{g}$. Moreover, it was revealed that observed by the local structure of the $\mathrm{Ag}^{+}$ion, silver metals were existed in $\mathrm{Ag}$-Y-zeolite bulk prepared by HHP. Using HHP methods, the solidification of other zeolites, such as MFI and MOR etc, have also confirmed. Therefore, this HHP is significantly applicable to solidify many porous materials like zeolites and mesoporous materials with high surface area. Successful synthesis of dense and bulky Ag-Y-zeolite with significantly high surface areas by HHP method will lead to new applications as efficient photocatalysts for the decomposition of $\mathrm{NO}_{\mathrm{x}}\left(\mathrm{NO}\right.$ and $\left.\mathrm{N}_{2} \mathrm{O}\right)$ into $\mathrm{N}_{2}$ and $\mathrm{O}_{2}$.

\section{References}

1) P. A. Jacobs, J. B. Uytterhoeven, and H. K. Beyer, J.Chem. Soc., Chem. Commun. 1997, 128-129.

2) G. Calzaferri, S. Hug, T. Hugentobler, and B. Sulzberger, J.Photochem. 26, 109-118 (1984).

3) G. A. Ozin, and F. Hugues, J. Phys. Chem. 86, 5174-5179 (1982).

4) M. Anpo, M. Matsuoka, and H. Yamashita, Catal. Today. 35, 177-181 (1997).

5) Y. Kim and K. Seff, J. Am. Chem. Soc. 99, 7055-7057 (1977).

6) Y. Kim and K. Seff, J. Am. Chem. Soc. 100, 6989-6997 (1978).

7) L. B. McCusker, Ph. D. Thesis, University of Hawaii, 1980.

8) Y. Y. Huang, J. Catal. 32, $482-491$ (1974).

9) K. Tsutsumi, H. Takahashi, Bull. Chem. Soc. Jpn. 45, 2332-2337 (1972).

10) A. Kuperman, S. Nadimi, S. Oliver, G. A. Ozin, J. M. Garcés, and M. M. Olken, Nature, 365, 239-242 (1993).

11) N. Yamasaki, K. Yanagisawa, M. Nishioka, and S. Kanahara, J. Mater. Sci. Lett. 5, 355-356 (1986).

12) A. Nakahira, S. Takezoe, and Y. Yamasaki, Chem. Lett. 33, 1400-1401 (2004). 